\title{
Effects of Comprehensive Nursing on Anxiety Score, Incidence of Pressure Ulcers and Nursing Satisfaction in Elderly Patients with Femoral Neck Fracture
}

\author{
Jiewen Yao*, Yanfei Li \\ Department of Spinal and Trauma, The First Affiliated Hospital of Jinan University, Guangzhou, China \\ Email address: \\ 397490326@qq.com (Jiewen Yao),973517560@qq.com (Yanfei Li) \\ ${ }^{*}$ Corresponding author
}

To cite this article:

Jiewen Yao, Yanfei Li. Effects of Comprehensive Nursing on Anxiety Score, Incidence of Pressure Ulcers and Nursing Satisfaction in Elderly Patients with Femoral Neck Fracture. American Journal of Nursing Science. Vol. 9, No. 4, 2020, pp. 211-214.

doi: 10.11648/j.ajns.20200904.17

Received: May 11, 2020; Accepted: May 27, 2020; Published: June 4, 2020

\begin{abstract}
Objective: To explore the effect of comprehensive nursing on anxiety score, incidence of pressure ulcers and nursing satisfaction in elderly patients with femoral neck fracture. Methods: A total of 110 elderly patients with femoral neck fractures admitted to our hospital from February 2019 to February 2020 were randomly selected and divided into a study group and acontrol group with 55 cases in each group. The patients in the latter group adopted the traditional nursing mode while the patients in the former group mainly adopted the comprehensive nursing mode in a timely manner. During the observation of the nursing results of patients, the Harris score of hip function was used to evaluate the postoperative nursing effect and an obvious result was gotten. Besides, the self-rating anxiety scale was used to effectively evaluate the psychological state of patients. The occurrence of pressure ulcers was observed, and the specific nursing conditions of the two groups of patients were effectively compared and analyzed. Results: The Harris score of hip function and anxiety score of patients in the study group before surgery and at the time of discharge were more significant than those in the control group, and the incidence of complications was significantly lower than those in the control group $(\mathrm{P}<0.05)$. The incidence of pressure ulcers in the study group was $2.07 \%$ and $14.57 \%$ in the control group. It is easy to see that the incidence of pressure ulcers in the study group was significantly lower than that in the control group, and the difference between the two groups was statistically significant $(\mathrm{P}<0.05)$. Conclusion: The comprehensive nursing method for elderly patients with femoral neck fracture exerts a beneficial effect on joint function and psychological rehabilitation of patients after femoral neck surgery, and the incidence of pressure ulcers is obviously reduced.
\end{abstract}

Keywords: Comprehensive Nursing, Elderly Patients with Femoral Neck Fracture, Anxiety Score, Incidence of Pressure Ulcers, Nursing Satisfaction

\section{Introduction}

It is undeniable that the physical function of elderly patients continues to decline, and their skin elasticity is gradually insufficient. At the same time, they might have osteoporosis and these patients are more prone to have fractures under the action of external forces. After the patient's fracture occurs, their bone needs special treatment such as fixation, traction and so on. During the treatment, the patient needs to be in bed for long-term recuperation [1-2]. In addition, due to the relatively poor physical nutrition of elderly patients, the long-term compression of the patients in bed is more obvious, making them more likely to have pressure ulcers, which has an increased impact on the patient's physical pain and also increases thepatient's financial burden of treatment, leading to an adverse effect on the patient's quality of life. In more serious cases, it can cause the occurrence of sepsis and other diseases in the patient, leading them to death [3-4]. What is more, it also increases the workload of nursing staff as well as the contradiction between nurses and patients. Therefore, the incidence of pressure ulcers also gradually become the main index in the 
evaluation of the clinical nursing of patients. In this study, a total of 110 elderly patients with femoral neck fractures treated in our hospital from February 2019 to February 2020 were selected to observe and analyze the anxiety score, incidence of pressure ulcers and nursing satisfaction in elderly patients with femoral neck fractures. The report is now as follows.

\section{Materials and Methods}

\subsection{General Data}

A total of 110 elderly patients with femoral neck fracture surgery admitted to our hospital from February 2019 to February 2020 were randomly selected. These patients were mainly divided into groups, namely, a study group and a control groupwith 55 patients in each group. There are 52 male patients and 58 female patients and their age is mainly between 60 and 82 years old and the average age of the patients is $71.26( \pm 11.12)$ years old. Among them, the main complications of patients are diabetes, hypertension, heart disease and other diseases, all of these patients did not have pressure ulcers before entering the hospital for treatment. Furthermore, the patients need to take bed rest during hospitalization, and their daily life needs care by the nursing staff. The general data of the two groups were not statistically significant $(\mathrm{P}>0.05)$, and the two groups were comparable.

\subsection{Method}

\subsubsection{Control Group}

During the treatment of patients, the patients in the control group were mainly cared by conventional nursing methods.

\subsubsection{Study Group}

The patients in the control group were mainly cared with the use of comprehensive nursing method, which primarily includes ward rounding treatment for patients according to the specific situation of the nursing level. Besides, the nursing staff should observe the specific changes of the patient's condition effectively according to the doctor's advice, turn the patient over on time, and massage the patient's skin of the pressurized part as necessary. Furthermore, the bed sheets of the patient during hospitalization should be clean and dry, andthe patient's clothing need changing in time.

\section{(i). Diet Care}

In the process of patient care, it is necessary to carry out dietary intervention on the patient. In addition to observing the patient's taste and basic disease conditions, the patient's diet should be reasonably guided. For instance, the nursing staff should encourage the patients who need high-protein foodsto eat fish, meat and other foods, and make themeat enough fresh vegetables and fruits. As for the care of diabetic patients, they need sugar-free vegetables and fruits. Moreover, hypertensive patients are not allowed to eat preserved food. What is more, patients with malnutrition and severe weight loss need to take human blood albumin or amino acids and other nutrients according to the doctor's requirements, so as to promote the effective maintenance of the patient's bodily needs.

\section{(ii). Psychological Nursing}

When carrying out comprehensive care for patients, it is also imperative to provide psychological care as well as love for the patients, especially among the elderly patients who are more prone to unhealthy emotions and psychological burdens. If a negative impact has been made on the treatment of patients, it is more likely to cause pressure ulcers for them. Thus, nursing staff would better communicate actively with patients and their families to master the effective methods for preventing pressure ulcers, so that the incidence of disputes between nurses and patients continues to decrease.

(iii). Pressure Ulcer Care

The effective measures to prevent pressure ulcers are mainly to use air mattresses, and to reduce the shear force between the skin of the patientsand the mattress as much as possible. For patients with high-risk conditions in which pressure ulcers occur, it is imperative to perform skin handover treatment for the patients. What is more, a turn-over card needs to be established for the patientsso as to ensure that the nursing staff can assist the patient's turn-over every 2 hours. When congestion occurs in the patient's compressed area, no massage is needed so as to prevent deep tissue damage caused by massage.

(iv). Discharge Guidance

It is also imperative to strengthen the guidance of the patient's discharge when the patient is discharged from the hospital. Usually, it takes a long time for the patients with fractures to recover from the disease, and the patients are also prone to inactivity after they are discharged. In this case, the nursing staff should be able to actively guide the patients and their families to pay attention to the precautions after the patients are discharged from the hospital. In addition, nursing staff should advise patients and their families to review regularly so as to prevent patients from suffering from pressure ulcers and other related diseases.

\subsection{Statistical Methods}

SPSS18 statistical software was used for statistical analysis and processing of the related data, and the difference was statistically significant $(\mathrm{P}<0.05)$.

\section{Results}

The Harris score of hip function as well as anxiety score ofpatients in the study group before surgery and at the time of dischargewere more significant and the incidence of complications was significantly lower than that in the control group. The difference between the two groups was statistically significant $(\mathrm{P}<0.05)$. What is more, the incidence of pressure ulcers in the study group was significantly lower than that in the control group $(\mathrm{P}<0.05)$. The nursing satisfaction of the patients in the study group was significantly higher than that in the control group, and the difference between the two groups was statistically significant $(\mathrm{P}<0.05)$. In addition, the anxiety scores of the patients in the study group were lower than those 
in the control group, and the difference between the two

groups was statistically significant $(\mathrm{P}<0.05)$.

Table 1. Comparison of anxiety score, incidence of pressure ulcers and nursing satisfaction between the two groups.

\begin{tabular}{lllll}
\hline Group & Number of cases & Anxiety score (point) & Pressure ulcer incidence & Nursing satisfaction \\
\hline Study group & 55 & $30.02 \pm 6.04$ & $2.07 \%$ & $95.93 \%$ \\
Control group & 55 & $42.91 \pm 6.54$ & $14.57 \%$ & $77.54 \%$ \\
P & 1 & $<0.05$ & $<0.05$ & $<0.05$ \\
\hline
\end{tabular}

Table 2. Comparison between length of stay in hospital and Harris score of hip joint function.

\begin{tabular}{llll}
\hline Group & Number of cases & Length of stay (d) & Harris score of hip joint function \\
\hline Study group & 55 & $17.82 \pm 5.84$ & $65.56 \pm 8.84$ \\
Control group & 55 & $22.39 \pm 6.56$ & $57.63 \pm 8.76$ \\
$\mathrm{P}$ & $/$ & $<0.05$ & $<0.05$ \\
\hline
\end{tabular}

\section{Discussion}

\subsection{Femoral Neck Fracture Disease}

Femoral neck fracture disease is a relatively common disease in traumatology orthopedics, which accounts for a large proportion of elderly fracture patients. This is mainly related to the decline in fracture food caused by osteoporosis in the elderly and the serious aging phenomenon. The incidence of femoral neck fractures also has a gradual upward trend. Surgical treatment is the main method in the treatment of diseases. However, it must also be clear that this treatment has a relatively great harm to the patients because the elderly with femoral neck fracture diseases require long-term bed rest after surgical treatment, which is not only prone to diseases such as urinary retention, urinary tract infections and deep vein thrombosis of the lower extremities, but also has a serious negative impact on the patient's daily life ability and quality of life. Therefore, the way to promoting the recovery of joint function of patients with femoral neck fracturesas soon as possible has a positive effect on effectively avoiding patients' adverse emotions [5-7].

\subsection{Main Causes of Pressure Ulcers}

The main reason for the formation of pressure ulcer is caused by the patient's long-term immobility in bed, which causes the patient's local skin and tissues to be compressed for a long time, resulting in the patient's ischemia and hypoxia. In severe cases, it will even cause necrosis [8-12].

\subsection{Clinical Nursing}

In the process of clinical care, the nursing staff need to perform regular patient turn-over treatment for the patient, and also need to avoid the situation of damage caused by the patient's skin dragging during the turn-over process [13-15]. Nursing staff need to explain the prevention methods of pressure ulcers to patients and their families, and give patients the necessary comprehensive evaluation work when they are admitted to the hospital. Once the training of nursing staff can be strengthened, better guidance can be provided to patients. After all, preventing the existence of pressure ulcers can constantly reduce the pain and financial burden of patients. During the clinical work, when the nursing staff assist the patient to turn over, it needs to be done in pairs to protect the patient, avoiding the unwillingness to cooperate with the nursing staff because of the fear of pain and slow movement after the operation. After a patient undergoes a long-term posture break, the skin damage may be caused if the patient does not receive guidance at the beginning of the activity. Hence, the nursing staff need to effectively convey the harm and prevention methods of pressure ulcers to the patients and their families. In addition, nursing staff should regularly carry out health promotion activities to promote family members and patients to actively participate in nursing activities so as to ensure that nursing work can be more comprehensive and free of loopholes.

Some nursing staff have the problem of not paying attention to basic nursing work and skin care work. Hence, nursing staff should also actively learn and constantly improve their professional qualities, effectively carry out a comprehensive evaluation of patients upon admission and determine the relevant work plan according to the specific situation. What is more, the department should also be able to regularly organize training for nursing staff, constantly help nursing staff acquire more new knowledge, strengthen their guidance on patients' turning over so as to prevent patients from developing pressure ulcers. By doing so, patients' pain and financial burden can be reduced, providing necessary support for patients' early recovery and quality of life guarantee [16-20]. At the same time, it is imperative to pay attention to the observation of patients' psychological changes, effectively carry out psychological counseling and related activities for patients, and promote the patients' treatment confidence. On the basis of encouragement and attention, patients can get better treatment and care, and their nursing satisfaction can be improved.

The results of the study showed that the Harris score of the hip function and the anxiety scoreof patients in the study group before surgery and at the time of discharge were more significant and the incidence of complications was significantly lower than those in the control group. The difference was statistically significant $(\mathrm{P}<0.05)$. In addition, the incidence of pressure ulcers in the study group during hospitalization was mainly $2.07 \%$ while $14.57 \%$ in the control 
group. It can be seen that the incidence of pressure ulcers in the study group was significantly lower than that in the control group. The difference between the two groups was statistically significant $(\mathrm{P}<0.05)$.

\section{Conclusion}

Based on the above discussion, comprehensive nursing care for elderly patients with femoral neck fractures can have a beneficial effect on the joint function and the psychological recovery of patients after femoral neck surgery and the incidence of pressure ulcers has a significant reduction, which is worthy of clinical promotion.

\section{References}

[1] Wu Zhifen. Application effect of comprehensive nursing in care of elderly patients with femoral neck fracture and coronary heart disease $[\mathrm{J}]$. Cardiovascular Disease Prevention and Treatment Knowledge (Academic Edition), 2020, 10 (04): 67-69.

[2] Zhang Yongjie. Observation on the application of comprehensive nursing in elderly patients with femoral neck fracture [J]. Electronic Journal of Clinical Medicine Literature, 2020, 7 (08): 108-109.

[3] Gong Qing. Study on the effect of comprehensive nursing on elderly patients with femoral neck fracture receiving surgical treatment $[\mathrm{J}]$. Journal of Contemporary Medicine, 2019, 17 (23): 197-198.

[4] Ren Yu. The effect of comprehensive nursing intervention on hip joint function, complications and daily living ability of elderly patients with femoral neck fracture [J]. Chinese Medical Guide, 2019, 17 (26): 267.

[5] Qian Jialan, Wang Wen. The effect of comprehensive nursing on elderly patients with femoral neck fracture after operation to prevent the occurrence of deep venous thrombosis of lower extremities [J]. Journal of Contemporary Medicine, 2019, 17 (16): 237-239.

[6] Li Chao. The effect of comprehensive nursing intervention on elderly patients with femoral neck fracture after operation $[\mathrm{J}]$. Modern Diagnosis and Treatment, 2019, 30 (11): 1957-1958.

[7] Chen Juan, Ruan Lili, Ye Yunqing. Application effect of comprehensive nursing in postoperative negative emotions of elderly patients with femoral neck fracture [J]. International Journal of Nursing, 2019, 38 (11): 1614-1616.

[8] Chen Juhong. Application effect analysis of comprehensive nursing intervention in artificial hip replacement for elderly femoral neck fracture $[\mathrm{J}]$. Practical Electronic Journal of Clinical Nursing, 2019, 4 (22): 9-10.
[9] Jiang Xuefang, Chen Lianmei. Comprehensive nursing intervention on postoperative joint function and pain in elderly patients with femoral neck fracture surgery [J]. Journal of Practical Clinical Nursing, 2019, 4 (20): $39+44$.

[10] Xu Xiyan, Wang Wei, Sun Xinhong, et al. Analysis of the value of comprehensive nursing in elderly femoral neck fractures and its impact on quality of life [J]. Electronic Journal of Clinical Medicine and Literature, 2019, 6 (22): 143.

[11] Fan Hongyan. Observation of the clinical effect of comprehensive nursing combined with total hip arthroplasty in the treatment of elderly femoral neck fractures $[\mathrm{J}]$. Contemporary Nurses (Early Issue), 2019, 26 (01): 63-65.

[12] Li Suping, Yan Juanchun, Huang Mulan. Comprehensive nursing intervention analysis of artificial femoral head replacement for elderly femoral neck fractures [J]. Sichuan Journal of Anatomy, 2018, 26 (04): 75-77.

[13] Zhang Ning, Liu Qing, Xie Fan, et al. Analysis of the effect of comprehensive nursing on elderly patients with femoral neck fracture undergoing artificial total hip replacement [J]. Contemporary Medicine Journal, 2018, 16 (22): 276-277.

[14] Guo Xiurong, Ye Qing. Analysis on the application effect of comprehensive high-quality nursing in elderly patients with femoral neck fractures [J]. Modern Practical Medicine, 2018, 30 (09): 1251-1252 + 1259 .

[15] Sun Li. Observation on the clinical effect of comprehensive nursing combined with total hip arthroplasty in the treatment of elderly femoral neck fractures [J]. Journal of Practical Clinical Nursing, 2018, 3 (34): 45-46.

[16] Liu Xiaohui. The effect of comprehensive nursing on elderly patients with femoral neck fracture on their postoperative pain, anxiety and depression [J]. Journal of Contemporary Medicine, 2018, 16 (15): 276-277.

[17] Zhao Shanni, Liu Juan, Wang Juan. The effect of comprehensive nursing intervention on postoperative pain in elderly patients with femoral neck fracture [J]. China Health Standards Management, 2018, 9 (14): 158-159.

[18] Zhang Qingfen. Effect analysis of comprehensive nursing on postoperative pain, anxiety and depression in elderly patients with femoral neck fracture [J]. Chinese Community Physician, 2018, 34 (19): 149-150.

[19] Zhao Tongjie. Comprehensive nursing application for elderly patients with femoral neck fracture [J]. The World 's Latest Medical Information Digest, 2018, 18 (32): 238-239.

[20] Tian Hexiao, Yin Feige, Wang Gonglei. Effect of Xuefu Zhuyu Decoction combined with comprehensive nursing on prevention of deep venous thrombosis of lower extremity after femoral neck fracture [J]. Haixia Pharmaceutical, 2018, 30 (02): $217-219$. 